\title{
High-speed holographic imaging using compressed sensing and phase retrieval
}

\section{Zihao Wang, Donghun Ryu, Kuan He, Roarke Horstmeyer, Aggelos Katsaggelos, et al.}

Zihao Wang, Donghun Ryu, Kuan He, Roarke Horstmeyer, Aggelos Katsaggelos, Oliver Cossairt, "High-speed holographic imaging using compressed sensing and phase retrieval," Proc. SPIE 10222, Computational Imaging II, 102220G (1 May 2017); doi: 10.1117/12.2262737

SPIE Event: SPIE Commercial + Scientific Sensing and Imaging, 2017, Anaheim, California, United States 


\title{
High-speed holographic imaging using compressed sensing and phase retrieval
}

\author{
Zihao Wang ${ }^{\mathrm{a}}$, Donghun Ryu ${ }^{\mathrm{b}}$, Kuan $\mathrm{He}^{\mathrm{a}}$, Roarke Horstmeyer ${ }^{\mathrm{c}}$, Aggelos Katsaggelos ${ }^{\mathrm{a}}$, and \\ Oliver Cossairt ${ }^{\mathrm{a}}$ \\ ${ }^{a}$ EECS* Northwestern University, Evanston, IL 60208, USA \\ ${ }^{b}$ Electrical Engineering, California Institute of Technology, Pasadena, CA 91125, USA \\ ${ }^{\mathrm{c} C h a r i t e ́ ~ M e d i c a l ~ S c h o o l, ~ H u m b o l d t ~ U n i v e r s i t y ~ o f ~ B e r l i n, ~ B e r l i n ~ 10117, ~ G e r m a n y ~}$
}

\begin{abstract}
Digital in-line holography serves as a useful encoder for spatial information. This allows three-dimensional reconstruction from a two-dimensional image. This is applicable to the tasks of fast motion capture, particle tracking etc. Sampling high resolution holograms yields a spatiotemporal tradeoff. We spatially subsample holograms to increase temporal resolution. We demonstrate this idea with two subsampling techniques, periodic and uniformly random sampling. The implementation includes an on-chip setup for periodic subsampling and a DMD (Digital Micromirror Device) -based setup for pixel-wise random subsampling. The on-chip setup enables direct increase of up to 20 in camera frame rate. Alternatively, the DMD-based setup encodes temporal information as high-speed mask patterns, and projects these masks within a single exposure (coded exposure). This way, the frame rate is improved to the level of the DMD with a temporal gain of 10 . The reconstruction of subsampled data using the aforementioned setups is achieved in two ways. We examine and compare two iterative reconstruction methods. One is an error reduction phase retrieval and the other is sparsity-based compressed sensing algorithm. Both methods show strong capability of reconstructing complex object fields. We present both simulations and real experiments. In the lab, we image and reconstruct structure and movement of static polystyrene microspheres, microscopic moving peranema, macroscopic fast moving fur and glitters.
\end{abstract}

Keywords: high-speed imaging, digital holography, compressed sensing, phase retrieval

\section{INTRODUCTION}

In recent years, we have witnessed a great interest in exploiting the redundant nature of signals. The redundancy of acquired signals provides the opportunity to sample data in a compressive approach. Candès et al. ${ }^{1,2}$ and Donoho ${ }^{3}$ have discussed the high probability of reconstructing signals with high fidelity from few random measurements, provided that the signals are sparse or compressible in a known basis. Since then, the theory of compressed sensing (CS) has been widely applied to computational imaging. Lustig et al. ${ }^{4}$ described the natural fit of CS to magnetic resonance imaging (MRI). Gan ${ }^{5}$ proposed block compressed sensing method for natural images, which is applicable for low-power, low-resolution imaging devices. Brady et al. ${ }^{6}$ showed that holography can be viewed as a simple spatial encoder for CS and demonstrated 3D tomography from 2D holographic data.

Gabor's invention of holography in $1948^{7}$ has provided an effective method for recording and reconstructing a 3D light field from a captured 2D hologram. The use of a CCD camera to digitally record holographic interference patterns has made digital holography (DH) an emerging technology with a variety of imaging applications, such as particle imaging, tracking in biomedical microscopy ${ }^{8-12}$ and physical process profiling and measuring. ${ }^{13-17}$ Digital Gabor/in-line holography (DIH) is a simple, lensless, yet effective setup for capturing holograms. The simplicity of DIH is balanced by the requirement that objects be small enough to avoid occluding the reference beam significantly. ${ }^{18}$ Extensive discussions and applications of DIH have been focused on microscopic imaging, i.e. small

\footnotetext{
*Electrical Engineering and Computer Science

Further author information: (Send correspondence to Z.W.)

Z.W.: E-mail: zwinswang@gmail.com

http: //compphotolab.northwestern.edu
}

Computational Imaging II, edited by A. Mahalanobis, A. Ashok, L. Tian, J. C. Petruccelli, K. S. Kubala,

Proc. of SPIE Vol. 10222, 102220G · @ 2017 SPIE · CCC code: 0277-786X/17/\$18 · doi: 10.1117/12.2262737 
and fast-moving objects. ${ }^{19-21}$ The tracking of fast movements usually entails multiple exposures. ${ }^{8,10,15,16,22,23}$ Temporal resolution is usually limited to the 10-100 millisecond range and little research has been conducted on temporal compression. However, in recent years, CS has proved a useful tool to increase the spatial information encoded in DH. ${ }^{6,24}$ Rivenson et al. ${ }^{25}$ discussed the application of CS to digital Fresnel holography. Liu et al. ${ }^{26,27}$ and Song et al. ${ }^{28}$ improved subpixel accuracy for object localization and enhanced spatial resolution (superresolution). Furthermore, CS theory has proven successful for recovering scenes under holographic microscopic tomography, ${ }^{29}$ off-axis frequency-shifting holography ${ }^{30}$ as well as millimeter-wave holography. ${ }^{31}$ Coded apertures have also been used together with CS to provide robust solutions for snapshot phase retrieval. ${ }^{32,33}$ In view of recent research in $\mathrm{CS}$ and $\mathrm{DH}$, several natural questions arise: Can we extend coded aperture to coded exposure? Can we exploit the unused pixels in exchange for increased temporal resolution? Since holography is naturally suitable for recovering depth information, a further research question is whether 4D space-time information can be extracted from 2D data employing the CS framework.

Similar discussions have been initiated in the incoherent imaging regime. Leveraging multiplexing schemes in the temporal domain, e.g. coded exposure, has been demonstrated as an effective hardware strategy for exploiting spatiotemporal trade-offs in modern cameras. High speed sensors usually require high light sensitivity and large bandwidth due to their limited on-board memory. In 2006, Raskar et al. ${ }^{34}$ pioneered the concept of coded exposure when he introduced the flutter shutter camera for motion deblurring. The technique requires knowledge of motion magnitude/direction and cannot handle general scenes exhibiting complex motion. Bub et al. ${ }^{35}$ designed a high speed imaging system using a DMD (digital micromirror device) for temporal pixel multiplexing. Gupta et al. ${ }^{36}$ showed how per-pixel temporal modulation allows flexible post-capture spatiotemporal resolution trade-off. Reddy et al. ${ }^{37}$ used sparse representations (spatial) and brightness constancy (temporal) to preserve spatial resolution while achieving higher temporal resolution. Liu et al. ${ }^{38}$ used an over-complete dictionary to sparsely represent time-varying scenes. Koller et al. ${ }^{39}$ discussed several mask patterns and proposed a translational photomask to encode scene movements extending the work of. ${ }^{40}$ These methods have proved successful for reconstructing fast moving scenes by combining cheap low frame-rate cameras with fast spatiotemporal modulating elements. While all of these techniques enable high speed reconstruction of $2 \mathrm{D}$ motion, incorporating holographic capture offers the potential to extend the capabilities to 3D motion. Moreover, in many holography setups, the energy from each scene is distributed across the entire detector so that each pixel contains partial information about the entire scene. This offers the potential for improved performance relative to incoherent architectures.

In this paper, we summarize two previous works. One exploits both spatial and temporal redundancy in natural scenes and generalizes to a 4D (3D positon with time) system model. ${ }^{41}$ We show that by combining digital holography and coded exposure techniques using a CS framework, it is feasible to reconstruct a 4D moving scene from a single $2 \mathrm{D}$ hologram. We demonstrate a temporal super resolution of $10 \times$. The second one focuses on lens-free on-chip imaging setup for in vivo scenes of biological samples. ${ }^{42}$

\section{SPACE-TIME COMPRESSIVE SAMPLING}

As illustrated in Fig. 1, consider a $4 \mathrm{D}$ field $V(x, y, z, t)$, which propagates along the positive $z$-direction. Along the propagation path, a high-speed coded mask $M(x, y, t)$ is located at $z_{1}$. A sensor is placed on the sensing plane $z_{2}$. In one frame, the sensor captures the intensity of the field during an exposure time of $\Delta t$. The volume can be discretized into $N_{d}$ planes, with the furthest plane having a distance of $d_{n}$ with respect to the observation plane at $z_{0}$. In Gabor holography, the object beam and the reference beam overlap with each other. This requires the objects to be sparse so that the occlusion of the reference beam is negligible. Under this assumption, the field $V$ in reality represents the summation of the object field $O$ and the constant reference field $R$. Thus, the field at $z_{0}$ is

$$
E_{0}\left(x, y, t ; z_{0}\right)=\sum_{n=1}^{N_{d}} H_{d_{n}} * O\left(x, y, z_{0}-d_{n}, t\right)+R,
$$

where $H_{d_{n}}=-\frac{i k}{2 \pi d_{n}} \exp \left[i k\left(x^{2}+y^{2}+d_{n}^{2}\right)^{\frac{1}{2}}\right]$ denotes the convolutional kernel for distance $d_{n}$. At the sensing 


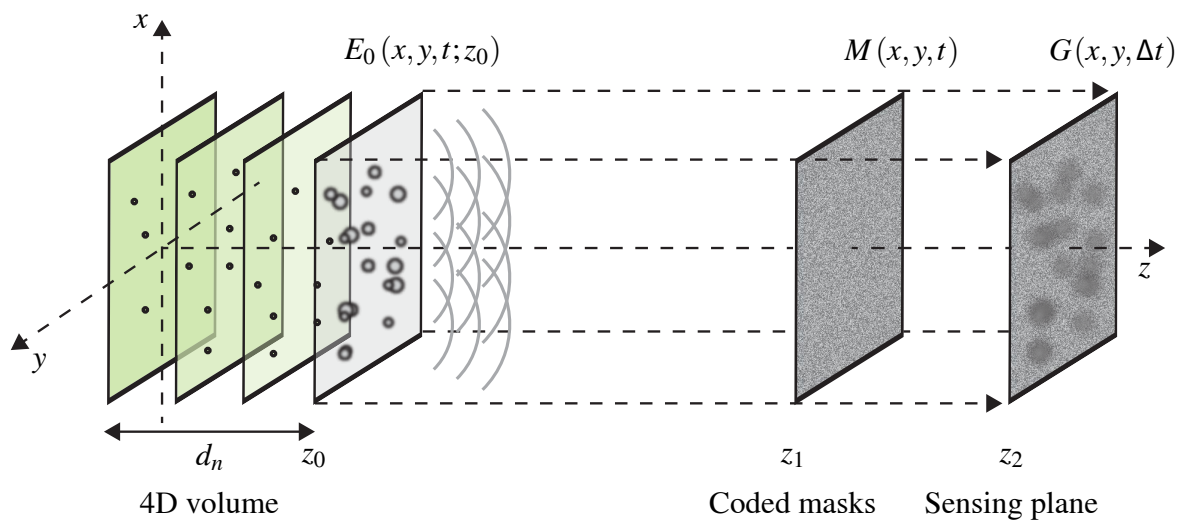

Figure 1. 4D holographic model. $E_{0}\left(x, y, t ; z_{0}\right)$ : projection of a $4 \mathrm{D}$ field at $z_{0}$, the $n$-th depth plane has a distance of $d_{n}$ to $z_{0} ; M(x, y, t)$ : temporal coded mask located at $z_{1} ; G(x, y, \Delta t)$ : captured image with an integral over $\Delta t$. The sensor is located at $z_{2}$.

plane, during one exposure time $\Delta t$, the sensed image can be expressed as an integral of the intensity $I$ of the field as

$$
\begin{aligned}
G(x, y, \Delta t) & =\int_{t=t_{0}}^{t_{0}+\Delta t} I\left(x, y, t ; z_{2}\right) d t \\
& =\int_{t=t_{0}}^{t_{0}+\Delta t}\left|H_{z_{2}-z_{1}} *\left\{M(x, y, t)\left[H_{z_{1}-z_{0}} * E_{0}\left(x, y, t ; z_{0}\right)\right]\right\}\right|^{2} d t
\end{aligned}
$$

where $H_{z_{1}-z_{0}}$ denotes the propagation process (convolutional kernel) from $z_{0}$ to $z_{1} ; H_{z_{2}-z_{1}}$ denotes the propagation process (convolutional kernel) from $z_{1}$ to $z_{2} ; M(x, y, t)$ denotes a time-variant mask located at $z_{1}$. Equation (2) describes the continuous form of the sensing process. However, the mask is operated in a discrete form at high frame rates. Suppose that for each sensor frame the coded mask changes $T$ times at equal intervals of $\tau=\Delta t / T$ during $\Delta t$. Then the discretized form of $G$ is

$$
\begin{aligned}
G(x, y, \Delta t) & =\sum_{i=0}^{T-1} \int_{t=t_{i}}^{t_{i+1}}\left|H_{z_{2}-z_{1}} *\left\{M(x, y, t)\left[H_{z_{1}-z_{0}} * E_{0}\left(x, y, t ; z_{0}\right)\right]\right\}\right|^{2} d t \\
& =\tau \sum_{i=0}^{T-1}\left|H_{z_{2}-z_{1}} *\left\{M\left(x, y, t_{i}\right)\left[H_{z_{1}-z_{0}} * E_{0}\left(x, y, t_{i} ; z_{0}\right)\right]\right\}\right|^{2} \\
& =\tau \sum_{i=0}^{T-1}\left|H_{z_{2}-z_{1}} *\left\{M\left(x, y, t_{i}\right)\left[H_{z_{1}-z_{0}} *\left(\sum_{n=1}^{N_{d}} H_{d_{n}} * O\left(x, y, z_{0}-d_{n}, t_{i}\right)+R\right)\right]\right\}\right|^{2} \\
& =\tau \sum_{i=0}^{T-1}\left|O_{c, i}+R_{c, i}\right|^{2},
\end{aligned}
$$

where we denote $O_{c, i}$ and $R_{c, i}$ as the transformed field at the capture plane $z_{2}$ for each time frame $i$.

Then the captured intensity term $I$ can be expanded as $I=\left|O_{c}+R_{c}\right|^{2}=O_{c} \cdot R_{c}^{*}+O_{c}^{*} \cdot R_{c}+O_{c}^{2}+R_{c}^{2}$. (Time frame notation $i$ is omitted here.) In, ${ }^{6}$ Brady et al. neglected the nonlinearity imposed by the squared magnitude and considered the two terms $O_{c}^{2}+R_{c}^{2}$ (often referred to as noise and zero-order/DC term) as noise in the measurement model showing that they can be eliminated algorithmically using a CS reconstruction algorithm. In this work, we follow the same approach and the measured intensity can be expressed as

$$
I=\left\{O_{c} \cdot R_{c}^{*}+O_{c}^{*} \cdot R_{c}\right\}+O_{c}^{2}+R_{c}^{2}=2 \operatorname{Re}\left\{O_{c} \cdot R_{c}^{*}\right\}+E_{E}
$$


where $E_{E}$ combines $O_{c}^{2}$ and $R_{c}^{2}$ into a single term considered as error. We may further assume the reference to be 1 without loss of generality. Then the intensity term can be written as $I=2 R e\left\{O_{c}\right\}+E_{E}$. In experiment, we approximate the error term by recording the background image and subtract the scene image by this background for reconstruction.

Now we assume that the sensor pixels have the same dimensions as the mask pixels, the unknown field $O$ will have spatial dimensions $N_{M_{x}} \times N_{M_{y}}$, depth dimension $N_{d}$ and temporal dimension $T$. Further, if we represent the convolutional operations in Eq. (3) as circulant matrices, we can obtain the following compact form

$$
\begin{aligned}
\mathbf{g} & =2 \mathbf{S}_{T} \operatorname{Re}\left\{\mathbf{H}_{T, z_{21}}\left\{\mathbf{M}_{T}\left[\mathbf{H}_{T, z_{10}}\left(\mathbf{H}_{T, d_{n}} \mathbf{o}\right)\right]\right\}\right\}+\mathbf{e}+\mathbf{n} \\
& =A(\mathbf{o})+\mathbf{e}+\mathbf{n},
\end{aligned}
$$

where $A(\cdot)$ describes the complete forward model. Specifically, $\mathbf{S}_{T}=\left[I_{0}, \ldots, I_{T-1}\right]$ represents summation over time, where $I_{i}, i=0, \ldots, T-1$ is an identity matrix of size $\left(N_{M_{x}} \times N_{M_{y}}\right) \times\left(N_{M_{x}} \times N_{M_{y}}\right) ; M_{T}=$ $\operatorname{bldg}\left(M_{0}, M_{1}, \ldots, M_{T-1}\right)$ is a block diagonal matrix with each block $M_{i}$ being a diagonal matrix. Each diagonal element of $M_{i}$ represents the corresponding pixel operation, e.g. 0 or $1 ; \mathbf{H}_{T, z}=\left[\mathbf{H}_{0, z}, \mathbf{H}_{1, z}, \ldots, \mathbf{H}_{T-1, z}\right]$, where $z$ represents $z_{21}$ and $z_{10} \cdot \mathbf{H}_{i, z}$ is a circulant matrix with size $\left(N_{M_{x}} \times N_{M_{y}}\right) \times\left(N_{M_{x}} \times N_{M_{y}}\right)$ corresponding to the convolutional kernel $H(x, y, z)$. $\mathbf{H}_{T, d_{n}}=b l d g\left(\mathbf{H}_{0, d_{n}}, \mathbf{H}_{1, d_{n}}, \ldots, \mathbf{H}_{T-1, d_{n}}\right]$, where $\mathbf{H}_{i, d_{n}}=\left[\mathbf{H}_{i, 1}, \mathbf{H}_{i, 2}, \ldots, \mathbf{H}_{i, N_{d}}\right]$ represents the summation over depths.

In order to reconstruct the $4 \mathrm{D}$ volume, an optimization problem is formed as

$$
\hat{\mathbf{o}}=\underset{\mathbf{o}}{\operatorname{argmin}} \frac{1}{2}\|\mathbf{g}-A(\mathbf{o})\|_{2}^{2}+\lambda \Phi(\mathbf{o})
$$

where $\lambda>0$ is a regularization parameter and $\Phi(\cdot)$ is a regularizer on the unknown $4 \mathrm{D}$ field $\mathbf{o}$.

Here, we employ Total-Variation (TV) as the regularization function defined as

$$
\Phi(\mathbf{o})=\|\mathbf{o}\|_{T V}=\sum_{t=0}^{T-1} \sum_{n=1}^{N_{d}} \sum_{x=1}^{N_{M_{x}}} \sum_{y=1}^{N_{M_{y}}}\left|\nabla(O)_{x, y, n, t}\right|,
$$

where we note here that $\mathbf{o}$ is the vectorized version of the unknown $4 \mathrm{D}$ object field $O: N_{M_{x}} \times N_{M_{y}} \times N_{d} \times T$. Equation (7) is a generalized 4D TV regularizer. However, the choice of regularizer may vary by different purposes of reconstruction and/or properties of scenes. In experiment, a 3D TV (x, y, n) is used for resolving depths (Section 3.2), i.e., $\Phi_{x, y, n}(\mathbf{o})=\sum_{n} \sum_{x} \sum_{y}\left|\nabla(O)_{x, y, n}\right|$. TV on temporal domain is included for recovering subtle movement (Section 3.3), i.e., $\Phi_{x, y, t, n}(\mathbf{o})=\sum_{n} \sum_{t} \sum_{x} \sum_{y}\left|\nabla\left(O_{n}\right)_{x, y, t}\right|$. Also note that independent regularization parameters may be chosen for the spatial $(\mathrm{x}, \mathrm{y}, \mathrm{n})$ and time $(\mathrm{t})$ dimensions. We used Two-step Iterative Shrinkage/Thresholding (TwIST) algorithm ${ }^{43}$ for reconstruction.

\section{4D IMAGING}

\subsection{Setup}

Figure 2 shows the schematic of the experimental setup. The illumination is produced by a diode laser powered by a pulse generator with wavelength of $532 \mathrm{~nm}$. The input beam is expanded and collimated by a neutral density (ND) filter and a collimating lens set (plano-convex lens, $300 \mathrm{~mm} / 35 \mathrm{~mm}=8.57$ magnification, ND filter omitted). In this setup, all the lens are from Thorlabs LSB04. A digital micromirror device (DMD) is used to perform pixel-wise temporal modulation of the light field, similar to. ${ }^{35}$ For our experiments, we used the DLP $®$ LightCrafter $4500^{\top \mathrm{T}}$ from Texas Instruments Inc. The light engine includes a 0.45 -inch DMD with $>$ 1million mirrors, each $7.6 \mu \mathrm{m}$, arranged in 912 columns by 1140 rows in a diamond pixel array geometry. ${ }^{44}$ The DMD is placed approximately $70 \mathrm{~mm}$ distance away from the objects. An objective lens (single lens with focal length of $125 \mathrm{~mm}$, aperture diameter of $2.54 \mathrm{~cm}$ ) is placed in front of the CMOS monochromatic sensor and well-aligned with the DMD so that it images the DMD plane onto the sensor. The lens introduces a quadratic 


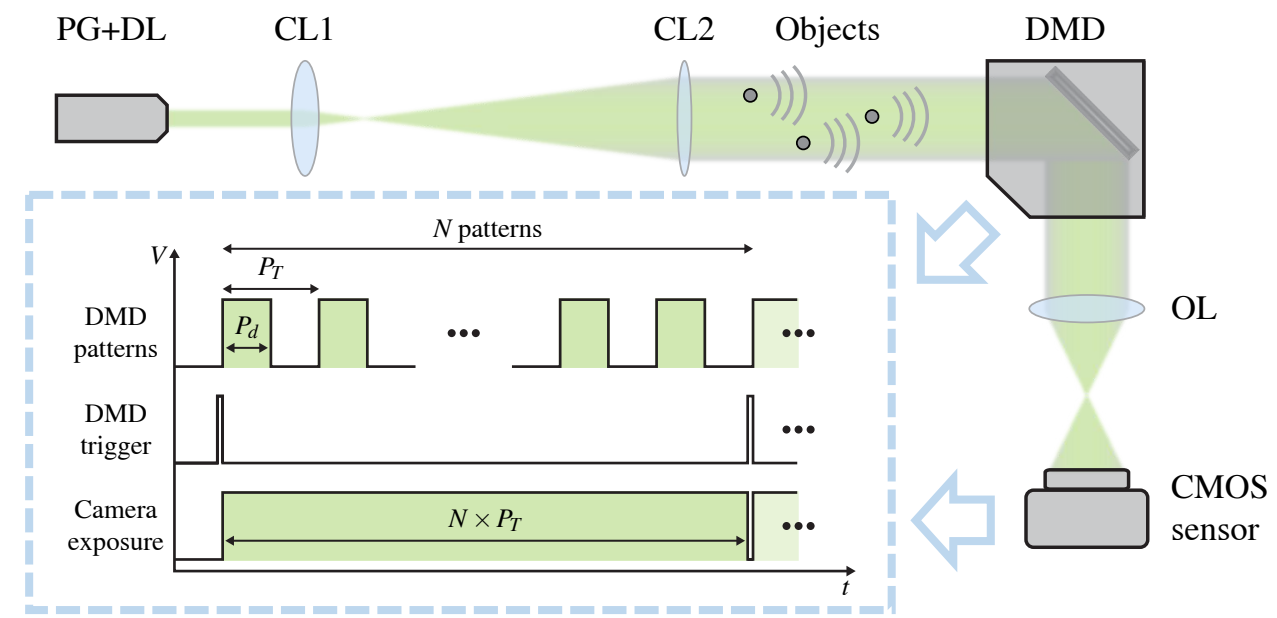

Figure 2. Schematic of the experimental setup. (PG: Pulse Generator; DL: Diode Laser; CL: Collimating Lens; DMD: Digital Micromirror Device; OL: Objective Lens. A trigger signal generated from the DMD is sent to the camera for exposure. The minimum time between successive DMD mask patterns is $P_{T}=500 \mu s$ with a pattern exposure $P_{d}=250 \mu s$. The camera is triggered every $\mathrm{N}$ patterns. ( $\mathrm{N}$ is equal to $\mathrm{T}$ in previous context.)

phase factor. Thus, if the sensor is placed a distance of $2 f$ from the OL, the phase is the same as $-2 f$ from the lens. In this way, $\mathbf{H}_{T, z_{21}}$ from Eq. (3) reduces to the identity matrix. We used a CMOS monochromatic sensor (Pointgrey GS3-U3-23S6M) with a resolution of $1920 \times 1200$ with a pixel pitch of $5.86 \mu \mathrm{m}$. The key factor is the synchronization between the DMD and the sensor. Each DMD pattern can be projected as fast as $P_{T}=500 \mu s$ with an effective pattern exposure of $P_{d}=250 \mu \mathrm{s}$. After N patterns are projected, a trigger signal is sent out to the camera which controls the shutter and results in a single exposure.

\subsection{Subsampling holograms}

We start our experiment by examining the reconstruction performance of subsampled holograms. Recovery of a 3D object field from a 2D hologram has been proposed in previous work. ${ }^{6}$ The recovery can be treated as inference of high-dimensional data from undersampled measurements. Figure 3 shows the experimental results of 3D recovery with pixel-wise subsampling. For this experiment, we captured two static fur placed a distance of $7.1 \mathrm{~cm}$ and $10.1 \mathrm{~cm}$ away from the DMD. Figure 3(a) shows the captured image. To preprocess the captured hologram, first we capture an image on the sensor with no object placed in the field of view - we refer to this as the background image. Note that this captured image corresponds to the term $R_{c}^{2}$ in Eq. (4). We then subtract the hologram by the background image, down-sampled to $960 \times 600$ and cropped the central $285 \times 285$ ROI around the object. Figure 3(b) shows the captured image of one pattern from the DMD. Each pattern randomly selects $10 \%$ of the entire image. To avoid aliasing artifacts caused by the diamond shaped sampling patterns on the DMD, we group together $4 \times 4$ adjacent pixels on the DMD to make a single superpixel. ${ }^{44}$ Since we are directly imaging on the DMD plane, the resolution is defined by the DMD. In our reconstructions, we design the regularizer to be $\Phi_{x, y, n}(\mathbf{o})=\sum_{n} \sum_{x} \sum_{y}\left|\nabla(O)_{x, y, n}\right|$, as our focus is the depth. In order to form the matrix A from Eq. (5), we capture images of the mask with no object present. These captured images are divided by the background image to remove the effect of beam non-uniformity. Figure 3(c) shows the subsampled hologram. Figure 3(d) and Fig. 3(e) compares reconstructions for the full hologram and subsampled hologram. The image $(285 \times 285)$ was reconstructed into a 3D volume $(285 \times 285 \times 120)$ with a depth range from $65 \mathrm{~mm}$ to $108 \mathrm{~mm}$. Shown are the images reconstructed at the depth planes corresponding to the location of the two hairs. In order to quantify the performance in terms of depth resolution, we used block variance ${ }^{45}$ for the edge pixel of the cross section by the two hairs. Higher variance infers higher contrast, and thus, higher resolution. The block variance was computed within a window of $21 \times 21$ pixels highlighted as blue and red in Fig. 3(d) and Fig. 3(e). Figure 3(f) shows the normalized variance versus depth from sensor. Two principle peaks are observed and can be inferred as the focus distance for the two furs. The peak around $d_{1}$ has strong signal in all four curves. This was because the object located there has larger size than the other one. As can be seen, using only $10 \%$ 


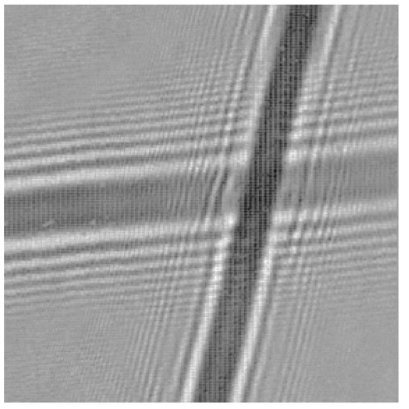

(a)

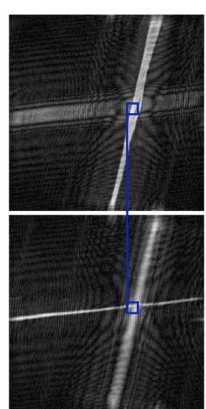

BP

(d) $100 \%$

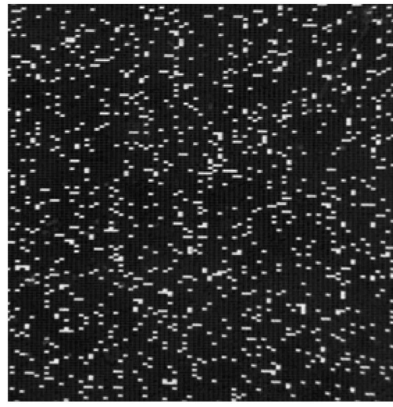

(b)

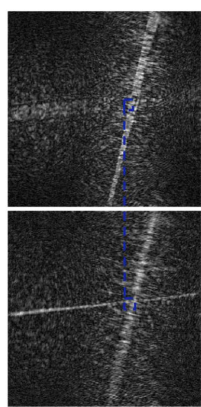

BP

(e) $10 \%$

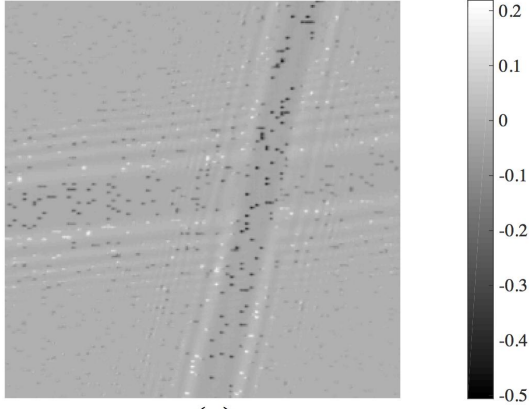

(c)

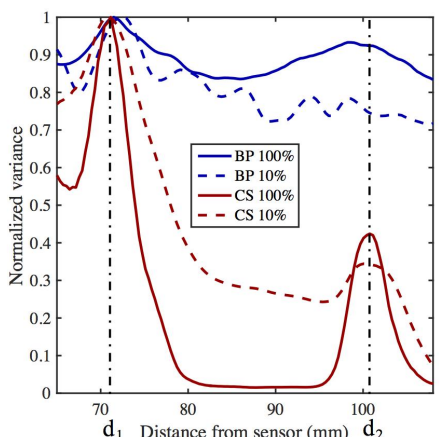

(f)

Figure 3. Subsampling holograms (background subtracted). (a)hologram of two static furs $7.1 \mathrm{~cm}$ and $10.1 \mathrm{~cm}$ away from sensor. (b) DMD mask, 10\%, uniformly random (background divided). (c) subsampled hologram. (d) Comparison of reconstructions from both back-propagation (BP) method and compressed sensing (CS) method using the full hologram. (e) Comparison for BP and CS using 10\% subsampled hologram. (f) Normalized variance vs. distance on z direction. Blue series: BP; red series: CS; full curve: 100\% hologram; dashed curve: $10 \%$ hologram.

of the data deteriorates both $\mathrm{BP}$ and $\mathrm{CS}$ reconstruction resolutions. And in $10 \%$ from $\mathrm{BP}$, it is even harder to track the second object because of the impact of mask pattern. This can also be observed in the left panel of Fig. 3(e) where the back propagation of the mask severely affected the objects. The variance decreases fast in CS reconstructions. This implies the denoising effect as well as the optical sectioning power of CS. In $10 \%$ reconstruction, the intermediate volume between the two objects were not denoised as good as in "100\%" case. This shows that greater subsampling factors reduce the effective depth resolution.

\subsection{Temporal multiplexing}

In the previous section, we analyzed the effect of subsampling on reconstruction performance for compressive holography. Here we show how to utilize the excess pixel bandwidth in the sensor to increase temporal resolution. Figure 4 shows a reconstruction result for dropping several flakes of glitter. The glitter flakes in Fig. 4(a) had size of $1 \mathrm{~mm}$ and were dropped in a range of $60 \mathrm{~mm}$ to $80 \mathrm{~mm}$ away from sensor. The glitter flakes were also blown by an air conditioner. Figure 4(b) shows the captured single image. Figure 4(c) shows preprocessed image which is subtracted by background image. In this case, the glitter flakes were moving at high speed. There was no overlap between two consecutive frames for the same flake. So each frame was recovered independently. For each frame, a depth range was estimated with 120 layers. The regularizer is designed to be $\Phi_{x, y, n}(\mathbf{o})=\sum_{n} \sum_{x} \sum_{y}\left|\nabla(O)_{x, y, n}\right|$. Figure $4(\mathrm{~d})$ shows a reconstruction map of 2 depths and 4 time frames. The downward and leftward motion of two glitter flakes can be observed. A similar refocusing method was used as in. ${ }^{45}$ Here, we scanned the reconstructed image by a $21 \times 21$ window and computed the variance (normalized) to get the focused depth information. If the normalized variance at defocused depth are higher than 0.5 , that pixel was rejected as background/noise. For adjacent pixels which have similar variance profile, the pixels were treated as a single particle. Figure 4(e) shows the normalized variance for two particles at $d_{1}$ and $d_{2}$. The particles are tracked at two locations pointed 

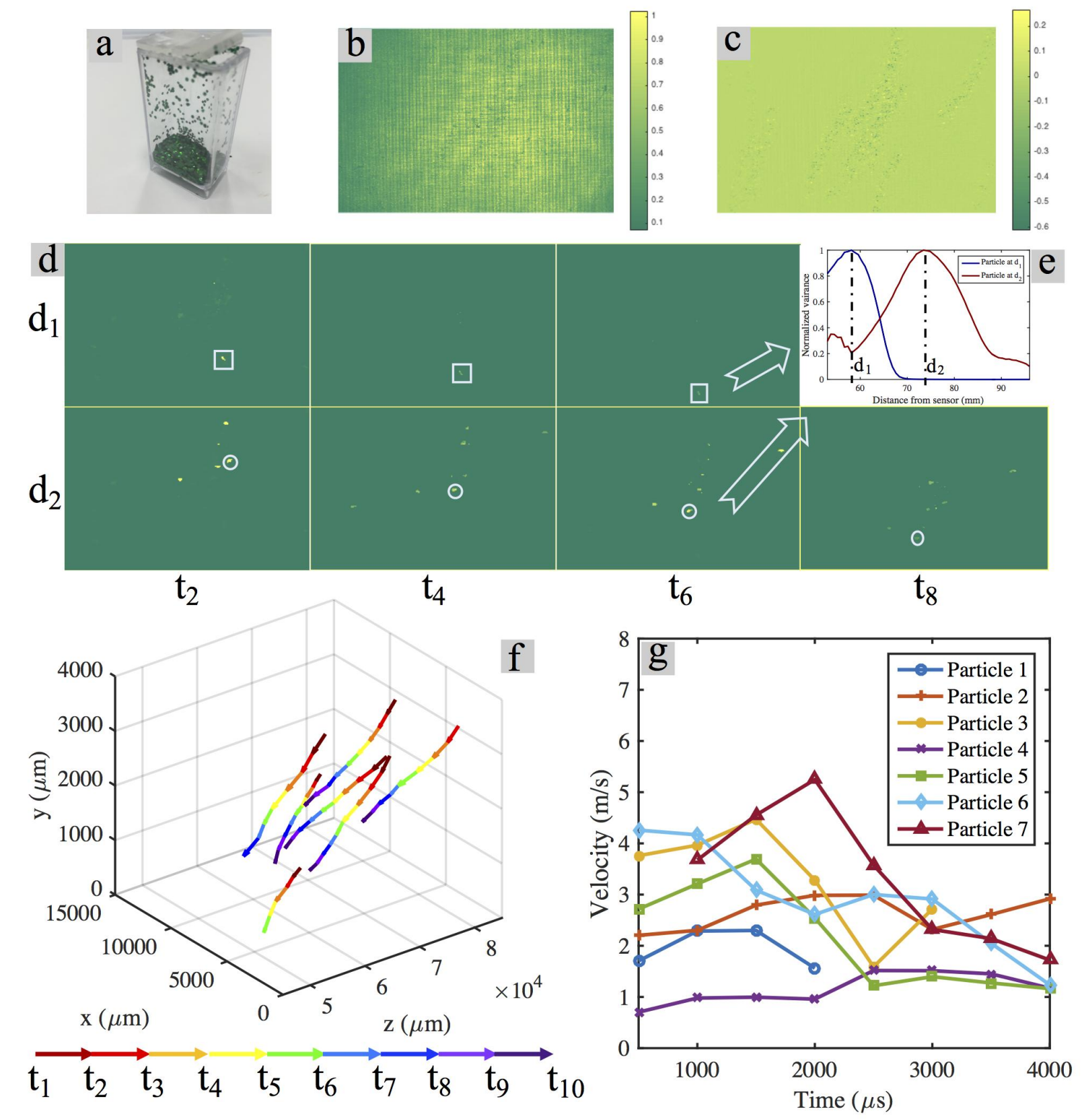

Figure 4. Reconstruction results from a single image (dropping glitters). (a) Glitters; (b) captured image; (c) normalized image; (d) reconstruction map. 2 depths and 4 out of 10 frames are shown; (e) normalized variance plot from 2 particles at $d_{1}$ and $d_{2}$; (f) 4D particle tracking; (g) velocity plotting with time range from $500 \mu s$ to $4000 \mu s$.

out by the arrows in Fig. 4(d). The overall tracking results are shown in Fig. 4(f) and Fig. 4(g). 7 particles are detected with $4 \mathrm{D}$ motion within $5 \mathrm{~ms}$. In Fig. 4(f), the temporal transition was represented by 10 different color arrows. Figure $4(\mathrm{~g})$ shows a velocity chart of the 7 particles. The velocity of each particle was computed by $v\left(t_{n}\right)=\left[d\left(t_{n+1}\right)-d\left(t_{n-1}\right)\right] / 2 \Delta t$, where $d\left(t_{n}\right)$ depicts the $3 \mathrm{D}$ location at $n$-th time frame, $\Delta t=500 \mu s$. The velocity of the particles ranges from $0.7 \mathrm{~m} / \mathrm{s}$ to $5.5 \mathrm{~m} / \mathrm{s}$. In this case, each time frame was processed separately. The data processing time for each frame takes about 7 hours for $A$ with the size of $(960 \times 600) \times(960 \times 600 \times 60)$. 


\section{LENS-FREE ON-CHIP IMAGING}

\subsection{Setup}

A simple schematic of an in-line holography setup is shown in Fig. 5(a). Here, we assume a distant point source illuminates a thin sample with a quasi-monochromatic, spatially coherent plane wave. While not done so here, it is direct to take into account the effects of partially coherent sample illumination. ${ }^{46}$ The optical field immediately after the sample, $f(x, y)=A(x, y) e^{i \phi(x, y)}$, offers a direct indication of its absorptivity within its amplitude $A(x, y)$, and optical thickness within its phase $e^{i \phi(x, y)}$.

The sample field $f(x, y)$ then propagates a distance $d$ to the detector plane, which contains an array of pixels. Directly above this plane, we denote the resulting complex field as $h\left(x_{a}, y_{a}\right)$, the hologram field, where $\left(x_{a}, y_{a}\right)$ are the spatial coordinates at this plane. Given sufficient distance between the sample and detector plane, it is possible to perform holography with a reference beam. In this work, we consider reference-free holographic imaging scenarios, which instead rely upon a phase retrieval algorithm to recover the complex field at the sample plane. In this reference-free phase retrieval, as commonly utilized in Coherent Diffraction Imaging, the detected intensity image is not necessarily regarded as hologram, instead as the intensity of a diffraction pattern ${ }^{47}$ Such a reference-free computational approach is common in other on-chip imaging setups.

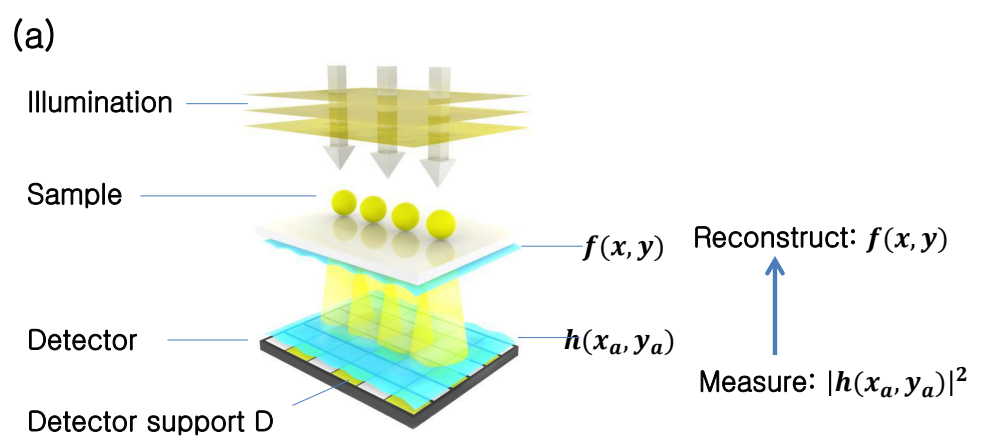

(b) $D=$ rectilinear sub-sampling

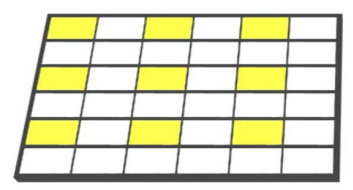

(c) $\mathrm{D}=$ random sampling

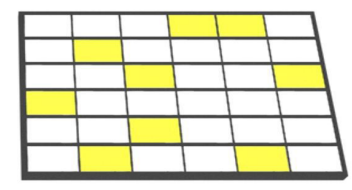

Figure 5. Subsampled phase retrieval (SPR) setup. (a) A detector array images the in-line hologram of a nearby complex sample, $f(x, y)$. The computational goal of "standard" lensless holography is to determine the complex sample $f$ given the measured hologram amplitudes, $|h|$, from every pixel int the detector array. SPR measures only a subset of values from the detector array (denoted in yellow), selected from (b) a spaced rectilinear grid or (c) randomly.

We may describe the diffraction of the sample field $f$ into the hologram $h$ using a propagation operator, $P_{d}[\cdot]$. Neglecting evanescent field effects and assuming this propagation is lossless, $P_{d}$ is invertible, and its inverse $P_{d}^{-1}[\cdot]$ represents time-reversed propagation from the detector plane back to the sample plane. The pixel array at the detector plane only detects the intensity of the hologram field:

$$
\left|h\left(x^{\prime}, y^{\prime}\right)\right|^{2}=\left|P_{d}[f(x, y)]\right|^{2},
$$

where $\left(x^{\prime}, y^{\prime}\right)$ are discretized versions of the detector plane coordinates $\left(x_{a}, y_{a}\right)$, making $\left|h\left(x^{\prime}, y^{\prime}\right)\right|^{2}$ a discrete function. We may assume the propagation operator $P$ also includes the effects of arbitrary pixel discretization. The goal of phase retrieval is to recover an accurate estimate of the complex sample transmission function, $f(x, y)$ from the measured set of intensities, $\left|h\left(x^{\prime}, y^{\prime}\right)\right|^{2}$. 


\subsection{Subsampled phase retrieval}

Phase retrieval algorithms compute the complex sample field from the measured diffraction pattern intensity through an iterative process. ${ }^{48}$ Here, we adopt the simple error reduction (ER) algorithm. ${ }^{48}$ It is also possible to use one of many other closely related strategies, ${ }^{49}$ including the hybrid input-output algorithm, or other more advanced solvers. ${ }^{50}$ Phase retrieval iteratively projects an initial estimate of $f$ onto two constraints in two different domains. In-line holography typically uses for its first constraint the object's support in the sample plane, and for its second constraint the measured hologram intensities in the detector plane.

\section{Phase retrieval algorithm for on-chip holography}

Sample plane

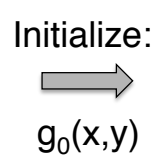

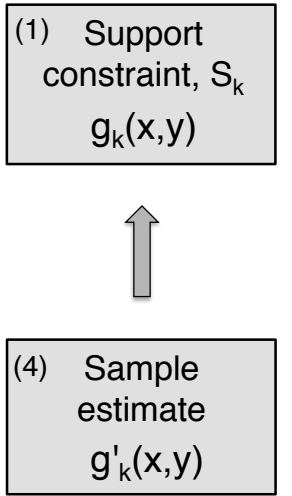
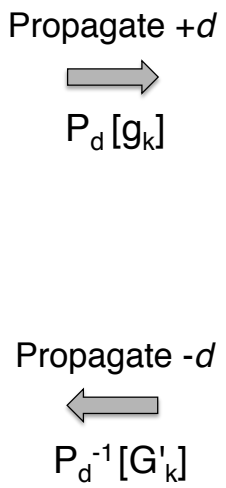

Detector plane

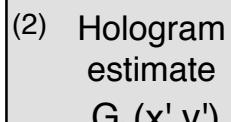

$\mathrm{G}_{\mathrm{k}}\left(\mathrm{x}^{\prime}, \mathrm{y}^{\prime}\right)$
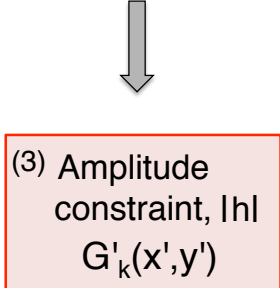

SPR: enforce only in subsampled set, $R$

Figure 6. Phase retrieval algorithm for on-chip holography. Each step is detailed in the text. Subsampling (SPR) only modifies the constraint in step 3. This modification results in over an order of magnitude potential speedup for lensless holographic video.

An outline of the phase retrieval algorithm for this "standard" case is diagrammed in Fig. 6. After initiating an initial complex sample estimate $g_{0}(x, y)$ at the sample plane, ER first digitally propagates it to the detector plane: $G_{k}\left(x^{\prime}, y^{\prime}\right)=P_{d}\left[g_{k}(x, y)\right]$. Here, $k$ denotes the $k$ th iterative loop, for $0 \leq k \leq n$ iterations. We use capital letters to denote our estimate at the detector plane, and lower case letters to denote it at the sample plane. We perform digital propagation using the angular spectrum method. Next, ER enforces the intensity constraint. It replaces the amplitudes of $G_{k}\left(x^{\prime}, y^{\prime}\right)$ with the experimentally measured amplitudes at the detector, $\left|h\left(x^{\prime}, y^{\prime}\right)\right|$ :

$$
G_{k}^{\prime}\left(x^{\prime}, y^{\prime}\right)=\left|h\left(x^{\prime}, y^{\prime}\right)\right| \frac{G_{k}\left(x^{\prime}, y^{\prime}\right)}{\left|G_{k}\left(x^{\prime}, y^{\prime}\right)\right|}, \forall\left(x^{\prime}, y^{\prime}\right) \in D
$$

where, in the standard PR case, $D$ represents the set of all pixels in the detector array and $G^{\prime}$ is the updated hologram estimate. We may equivalently represent this estimate update as, $G_{k}^{\prime}\left(x^{\prime}, y^{\prime}\right)=\left|h\left(x^{\prime}, y^{\prime}\right)\right| e^{i \phi_{k}\left(x^{\prime}, y^{\prime}\right)}$, which makes clear the intensity constraint step leaves the phase of the current hologram estimate, $\phi_{k}\left(x^{\prime}, y^{\prime}\right)$, unchanged. Third, ER propagates this intensity-constrained hologram estimate back to the sample plane: $g_{k}^{\prime}(x, y)=P_{d}^{-1}\left[G_{k}^{\prime}\left(x^{\prime}, y^{\prime}\right)\right]$. Fourth, ER applies a sample support constraint. It leaves unchanged all values within a defined subset of pixels, $S_{k}$, which typically represents the interior of a collection of cells or an organism of interest. However, it assumes that outside of this interior support area the sample exhibits a uniform absorptivity (i.e., the illumination light primarily passes to the detector unchanged). ER thus sets pixels outside of this 
support area to a uniform background value $b$ :

$$
g_{k+1}(x, y)= \begin{cases}g_{k}^{\prime}(x, y) & (x, y) \in S_{k} \\ b & (x, y) \notin S_{k}\end{cases}
$$

In this last step the iteration counter value $k$ increments for the next iteration. The above ER loop runs for a fixed number of $n$ iterations, or until some convergence criteria is satisfied. The complex algorithm output, $g_{n}(x, y)$, typically offers an accurate estimate of the amplitude and phase of the original optical field $f(x, y)$ at the sample plane.

Since we rarely know the exact support of each sample a-priori, we use two recent insights to ensure the constraint in Eq. (10) encourages successful algorithm convergence. First, we adaptively update the assigned background value, $b$, each iteration. Following, ${ }^{46}$ we set $b=\left\langle\left|g_{k}^{\prime}(x, y)\right|\right\rangle r(x, y) /\langle r(x, y)\rangle$ at iteration $k$, where \langle\rangle denotes the mean over all pixels and $r(x, y)$ is a fixed reference measurement formed by back-propagating a set of reference hologram amplitudes, $\left|h_{r}\left(x^{\prime}, y^{\prime}\right)\right|$ (which do not contain diffracted light from any cells or sample structure). The reference amplitudes $\left|h_{r}\right|$ may be acquired before the experiment, or simply selected from a region of the hologram where no sample structure is present.

Second, to improve the accuracy of Eq. (10), we also vary the set of pixels defining the sample support each iteration, $S_{k}$. We update $S_{k}$ with the "shrink-wrap" method. ${ }^{51}$ At a given iteration, this method first blurs and then thresholds the current sample estimate to form a new support boarder. Blurring helps smooth noise to regularize the support area, and also encourages algorithm stability. Unless otherwise stated, our shrink-wrap implementation uses a Gaussian blur kernel of $5^{2}$ pixels, a normalized threshold value of 0.15 , and updates the support every tenth iteration. The initial guess of the support follows the same routine.

\subsection{Experimental validation}

First, we verify the ability of SPR to measure quantitative phase by imaging a fixed sample of polystyrene microspheres $\left(30 \mu \mathrm{m}\right.$ in diameter, $n_{m}=1.5875$ refractive index, immersed in oil with $n_{o}=1.595$ refractive index). We first capture a full-resolution hologram of a large distribution of microspheres. One microsphere of interest, from a $300 \times 300$ pixel region, is shown in Fig. 7 (a). We digitally subsample this measured hologram in two different geometries (rectilinear and semi-random, as outlined in Section 2) at the following subsampling rates: $|D| /|R|=9,16$, and 25. Then, we input these subsampled images into our SPR algorithm and run $n=200$ iterations to recover the microsphere reconstructions shown in Fig. 7(b).

Although up to $96 \%$ of the original hologram image remains unused, SPR still accurately recovers the phase shift induced by each microsphere, as shown for example in Fig. 7(b). Here, we also attempt sample reconstruction after first performing cubic image interpolation on the sub-sampled holograms, which results in an unpredictable shift in the phase centroid. Finally, we quantify the accuracy of SPR by comparing its reconstructed phase to the known phase shift induced by an ideal sphere in Fig. 7(c1-c3). Here, we select the experimental phase shift values $\Delta \phi$ from along one row of pixels through the center of each sphere (dashed line). The known microsphere phase shift is determined by the optical path length difference of a wave passing through a $30 \mu \mathrm{m}$ circle with an index shift of $n_{m}-n_{o}=.0075$. From this experiment, we conclude that SPR maintains an accurate measure of quantitative phase.

Given quantitatively accuracy, we next use SPR with an in vivo biological specimen. A collection of Peranema, which are microorganisms that are primarily transparent and fall within the euglinoid family, are placed in medium onto a standard microscope slide. We position the slide at approximately $1910 \mu \mathrm{m}$ above the sensor and capture a series of holograms, a cropped example of which is shown in Fig. 8(a). First, we select a $300^{2}$ pixel region of the hologram and perform standard ER phase retrieval to reconstruct the sample amplitude and phase shown in Fig. 8(b)-(c).

For ground-truth comparison, we also place the same sample of Peranema beneath a 10X objective microscope and capture the intensity image in Fig. 8(d). Although the locations of each microorganism differ from those in reconstruction images due to their unpredictable movement, the structures of their main body qualitatively match. Two other qualitative points are worth noting: first, our reconstructed phase shows a clear boundary 


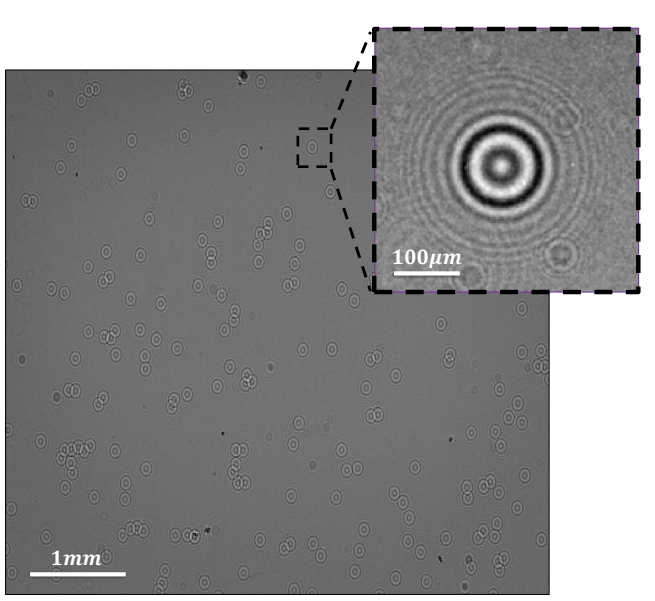

(a) Full field-of-view holograms

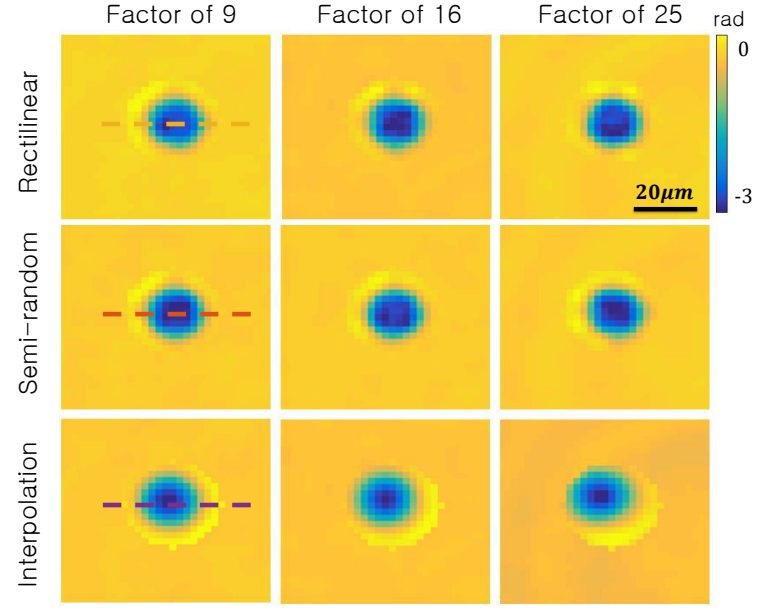

(b) Reconstructed phase

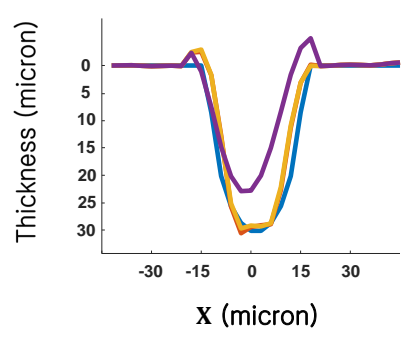

(c1) Factor of 9

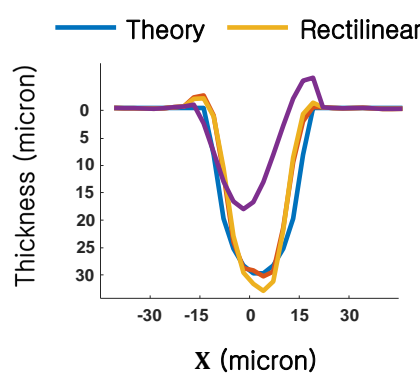

(c2) Factor of 16

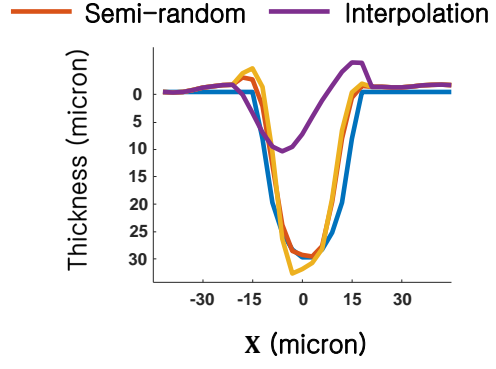

(c3) Factor of 25

Figure 7. Experimental results, on-chip imaging of polystyrene microspheres. (a) Raw detected hologram with one region of interest highlighted. (b) The recovered sample phase from the region of interest using the SPR algorithm (top and middle) and standard ER phase retrieval with interpolation (bottom). (c) Line traces through the center of the recovered microsphere phase (dashed lines) reveal quantitative agreement with the expected phase shift, even after reducing the number of pixels in factor of 9,16 and 25 . 
Full field-of-view holograms
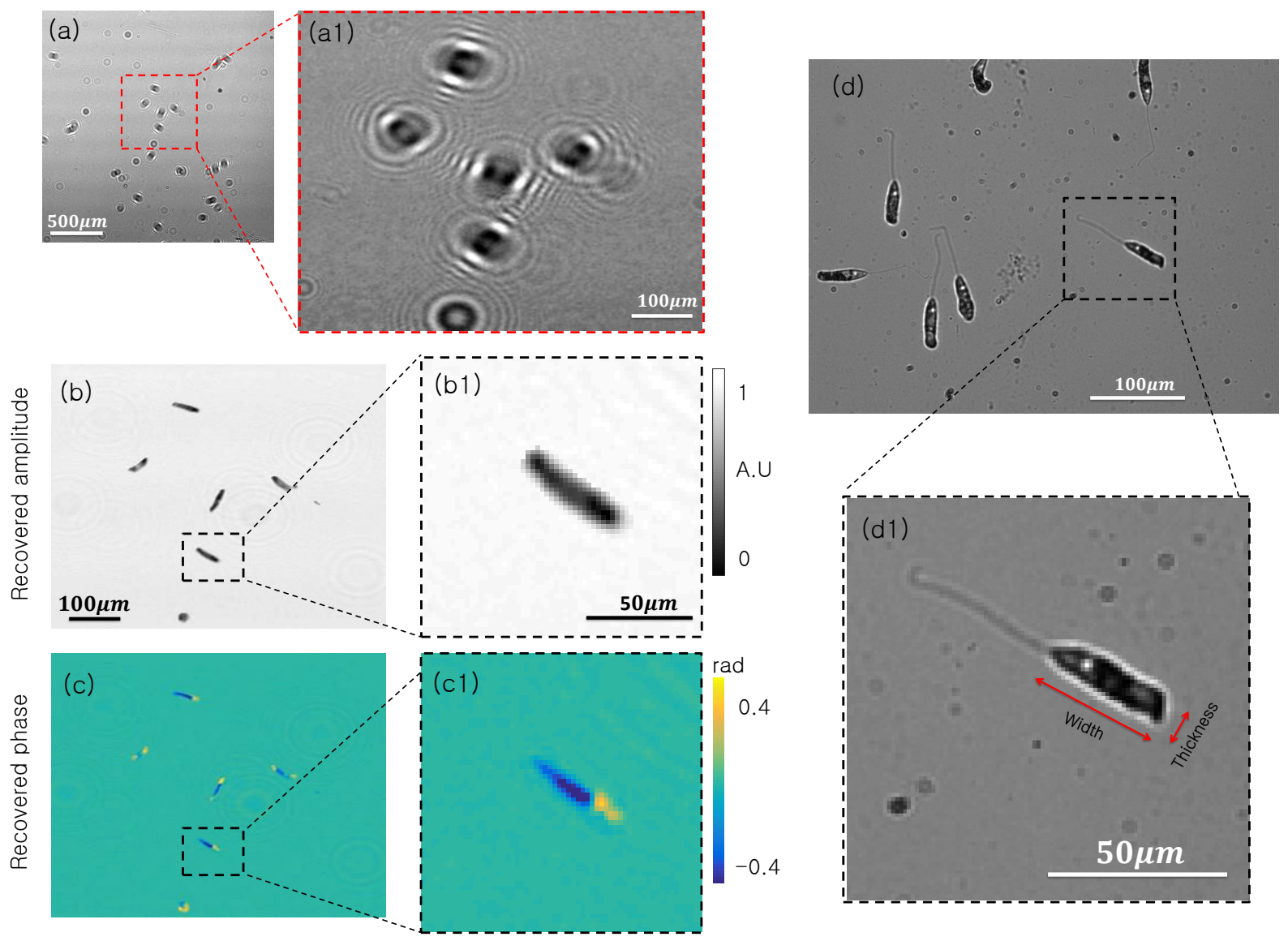

Figure 8. Experimental results for on-chip imaging of live peranema microorganisms. (a) Full field-of-view hologram. (a1) Inset of interest. (b) Reconstructed amplitude with standard ER phase retrieval. (b1) Recovered amplitude of single peranema. (c) Reconstructed phase with standard ER phase retrieval. (c1) Recovered phase of single peranema. (d) 10X microscope image of live peranema. (d1) Inset of single peranema offers a useful "ground truth" comparison. 
between the front and back section of each microorganism, consistent with the presence of their basal body. Second, our reconstructed images cannot resolve the microorganism flagellum (i.e., tail), which is primarily due to our system's limited resolution. We experimentally determined the tail width as approximately $2 \mu \mathrm{m}$, which is close to the $2.2 \mu \mathrm{m}$ pixel size of the digital sensor. Future experiments may resolve the flagellum by using a sensor with smaller pixels or with additional processing (see discussion section).

Next, we operate the second CMOS sensor which provides rectlinear subsampling mode to capture holographic movies of microorganisms moving over time. We test 3 different subsampling strategies: $|D| /|R|=1,4$, and 9 . For this particular sensor, these subsampled data rates correspond to the ability to increase the sensor frame rate by a factor of 1, 3.1 and 5.5, respectively (from 4.4 FPS for no subsampling to 24.8 FPS for $9 \times$ subsampling). For each frame, we apply the SPR algorithm to recover amplitude and phase of each Peranema across the entire sensor. Example insets of the recovered amplitude and phase of single Peranema are displayed in Fig. 9. Our subsampling strategy offers videos with much smoother motion between consecutive frames, which is not originally captured in full resolution reconstructions. Again, the support from subsampled holograms in each frame is generated by the modified shrink-wrap algorithm, which plays an important role in the holographic video reconstruction of the Peranema.

Finally, to quantitatively verify the accuracy of our SPR reconstructions, we compare the average width and thickness of a collection of Peranema bodies as measured from a single reconstructed frame (with $|D| /|R|=9$ ) to that measured from a standard microscope image. The mean value (MV) and standard deviation (SD) of the width of the Peranema from the SPR reconstructed frame is $50.137 \mu \mathrm{m}$ and $6.344 \mu$, respectively, which closely matches that from the microscope image $(\mathrm{MV}=47.818 \mu \mathrm{m}$ and $\mathrm{SD}=2.511 \mu \mathrm{m}$, width labeled in Fig. 8(d1)). Similarly, for the reconstructed thickness we have $\mathrm{MV}=11.579 \mu \mathrm{m}$ and $\mathrm{SD}=1.607 \mu \mathrm{m}$ for the $\mathrm{SPR}$ reconstruction, whereas the microscope image yields $\mathrm{MV}=12.937 \mu \mathrm{m}$ and $\mathrm{SD}=2.786 \mu \mathrm{m}$. Both width and thickness match within one standard deviation.

\section{CONCLUSION}

We have demonstrated two works on efficient space-time sampling setup to achieve high-speed imaging.

In the case of compressive holographic video, we use a DMD as a coded aperture that is imaged directly onto a sensor. While non-trivial to implement, in principle it is possible to fabricate a CMOS sensor with pixel-wise coded exposure control. The prototype showed that it is possible to simultaneously exceed the capture rate of imagers and recover multiple depths with reasonable depth resolution. In this paper, as an example, we presented a temporal increase factor of $10 \times$. A potential factor can be $24 \times$ based on the DMD we used. By means of spatio-temporal modulator, one is able to significantly increase the frame rate of the sensors. Based on this idea, the recovered frame rate is redefined by the modulator's frame rate. The coded-exposure technique enables high speed imaging with a simple frame rate camera. Digital in-line holography brings the capability of 3D tomographic imaging with simple experimental setup. Our Compressive Holographic Video technique is also closely related to phase retrieval problems commonly faced in holographic microscopy. Our space-time subsampling technique can be viewed as a sequence of coded apertures applied to a spatiotemporally varying optical field. In the future we plan to explore the connections between our CS reconstruction approach and the methods introduced in. ${ }^{32}$ In our general model, we place a coded aperture between the sensor and scene. In our prototype implementation we use a DMD as a coded aperture that is imaged directly onto a sensor. While not explored in this paper, we believe that adding defocus between the coded aperture plane and sensor may be beneficial for phase retrieval tasks, as in. ${ }^{32}$ In this work, we focus on a proof-of-principle demonstration of compressive holographic video. In the future, we hope to explore a diverse set of mask designs, as well as techniques for mask optimization.

In the case of lens-free on-chip imaging, we believe SPR offers a useful conceptual starting point for more advanced procedures. First, SPR currently does not consider the redundant nature of the video signal over time. Adopting the insights gained by SPR into a more general approach to optimize phase retrieval over both space and time will likely lead to additional video speedup. Methods such as optical flow may provide a good path forward in this regard. Second, SPR is capable of removing objects that are not in focus, which offers a means to simultaneously achieve optical sectioning. Third, the effectiveness of SPR indicates that it might also be useful for X-ray imaging and coherent diffraction imaging, as well as related techniques for ptychography. 


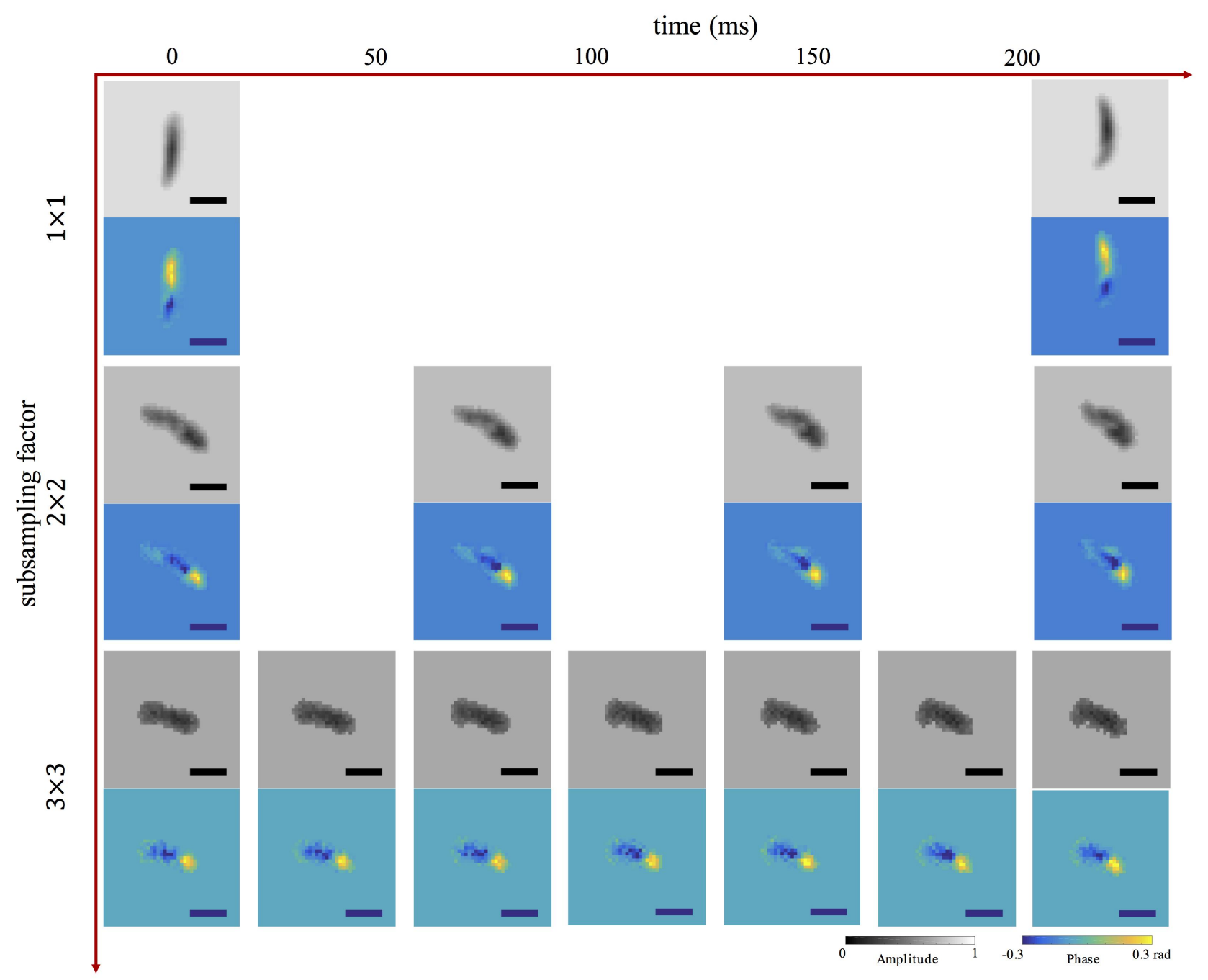

Figure 9. Example: subsampled holographic reconstruction of in vivo Peranema in motion (subsampling factor vs. time). Horizontal axis depicts time and vertical axis represents subsampling factor. Reconstructions of both amplitude and phase using all pixels on the detector are shown at top, while reconstructions from subsampled pixel array data, using a factor of 4 and 9, are in middle and bottom, respectively. Consecutive frames show Peranema motion from left to right. Frame rate: first row $-4.4 \mathrm{FPS}$, second row $-13.6 \mathrm{FPS}$, third row $-24.8 \mathrm{FPS}$. Scale bar is $22 \mu \mathrm{m}$. 


\section{ACKNOWLEDGMENTS}

This work was supported in part by National Science Foundation (NSF) CAREER grant IIS-1453192; Office of Naval Research (ONR) grant 1(GG010550)//N00014-14-1-0741; Office of Naval Research (ONR) grant \#N0001415-1-2735 and DARPA award (G001534-7510)//HR0011-16-C-0028.

\section{REFERENCES}

[1] Candès, E. J., Romberg, J., and Tao, T., "Robust uncertainty principles: exact signal reconstruction from highly incomplete frequency information," IEEE Trans. Inform. Theory 52, 489 - 509 (Feb. 2006).

[2] Candès, E. J. and Tao, T., "Near-optimal signal recovery from random projections: Universal encoding strategies?," IEEE Trans. Inform. Theory 52, 5406-5425 (Dec. 2006).

[3] Donoho, D. L., "Compressed sensing," IEEE Trans. Inf. Theory, 52, 1289-1306 (Apr. 2006).

[4] Lustig, M., Donoho, D. L., Santos, J. M., and Pauly, J. M., "Compressed sensing MRI," IEEE Signal Process. Mag. 25, 72-82 (Mar. 2008).

[5] Gan, L., "Block compressed sensing of natural images," in [International Conference on Digital Signal Processing], 403-406 (July 2007).

[6] Brady, D. J., Choi, K., Marks, D. L., Horisaki, R., and Lim, S., "Compressive holography," Opt. Express 17, 13040-13049 (Jul. 2009).

[7] Gabor, D., "A new microscopic principle," Nature 161(4098), 777-778 (1948).

[8] Memmolo, P., Miccio, L., Paturzo, M., Caprio, G. D., Coppola, G., Netti, P. A., and Ferraro, P., "Recent advances in holographic 3D particle tracking," Adv. Opt. Photon. 7, 713-755 (Dec. 2015).

[9] Xu, L., Peng, X., Miao, J., and Asundi, A. K., "Studies of digital microscopic holography with applications to microstructure testing," Appl. Opt. 40, 5046-5051 (Oct 2001).

[10] Su, T.-W., Xue, L., and Ozcan, A., "High-throughput lensfree 3d tracking of human sperms reveals rare statistics of helical trajectories," Proceedings of the National Academy of Sciences 109(40), 16018-16022 (2012).

[11] Lü, Q., Chen, Y., Yuan, R., Ge, B., Gao, Y., and Zhang, Y., "Trajectory and velocity measurement of a particle in spray by digital holography," Appl. Opt. 48, 7000-7007 (Dec. 2009).

[12] Dixon, L., Cheong, F. C., and Grier, D. G., "Holographic deconvolution microscopy for high-resolution particle tracking," Opt. Express 19, 16410-16417 (Aug. 2011).

[13] Saxton, M. J. and Jacobson, K., "Single particle tracking: Applications to membrane dynamics," Annual Review of Biophysics and Biomolecular Structure 26, 373-399 (1997).

[14] Katz, J. and Sheng, J., "Applications of holography in fluid mechanics and particle dynamics," Annual Review of Fluid Mechanics 42, 531-555 (2010).

[15] Tian, L., Loomis, N., Domínguez-Caballero, J. A., and Barbastathis, G., "Quantitative measurement of size and three-dimensional position of fast-moving bubbles in air-water mixture flows using digital holography," Applied Optics 49(9), 1549-1554 (2010).

[16] Xu, W., Jericho, M. H., Kreuzer, H. J., and Meinertzhagen, I. A., "Tracking particles in four dimensions with in-line holographic microscopy," Opt. Lett. 28, 164-166 (Feb. 2003).

[17] Nilsson, B. and Carlsson, T. E., "Simultaneous measurement of shape and deformation using digital lightin-flight recording by holography," Opt. Eng. 39(1), 244-253 (2000).

[18] Kim, M. K., [Digital Holographic Microscopy], Springer (2011).

[19] Garcia-Sucerquia, J., Xu, W., Jericho, S. K., Klages, P., Jericho, M. H., and Kreuzer, H. J., "Digital in-line holographic microscopy," Appl. Opt. 45, 836-850 (Feb. 2006).

[20] Xu, W., Jericho, M. H., Meinertzhagen, I. A., and Kreuzer, H. J., "Digital in-line holography for biological applications," Proc. Natl. Acad. Sci. 98(20), 11301-11305 (2001).

[21] Yu, X., Hong, J., Liu, C., Cross, M., Haynie, D. T., and Kim, M. K., "Four-dimensional motility tracking of biological cells by digital holographic microscopy," Journal of Biomedical Optics 19(4), 045001 (2014).

[22] Salah, N., Godard, G., Lebrun, D., Paranthoën, P., Allano, D., and Coëtmellec, S., "Application of multiple exposure digital in-line holography to particle tracking in a Bénard-von Kármán vortex flow," Measurement Science and Technology 19(7), 074001 (2008). 
[23] Yu, X., Hong, J., Liu, C., and Kim, M. K., "Review of digital holographic microscopy for three-dimensional profiling and tracking," Opt. Eng. 53(11), 112306 (2014).

[24] Lim, S., Marks, D. L., and Brady, D. J., "Sampling and processing for compressive holography," Appl. Opt. 50, H75-H86 (Dec. 2011).

[25] Rivenson, Y., Stern, A., and Javidi, B., "Compressive fresnel holography," J. Display Technol. 6, 506-509 (Oct. 2010).

[26] Liu, Y., Tian, L., Lee, J. W., Huang, H. Y. H., Triantafyllou, M. S., and Barbastathis, G., "Scanning-free compressive holography for object localization with subpixel accuracy," Opt. Lett. 37, 3357-3359 (Aug. 2012).

[27] Liu, Y., Tian, L., Hsieh, C.-H., and Barbastathis, G., "Compressive holographic two-dimensional localization with 1/30 2 subpixel accuracy," Optics express 22(8), 9774-9782 (2014).

[28] Song, J., Swisher, C. L., Im, H., Jeong, S., Pathania, D., Iwamoto, Y., Pivovarov, M., Weissleder, R., and Lee, H., "Sparsity-based pixel super resolution for lens-free digital in-line holography," Scientific reports 6 (2016).

[29] Hahn, J., Lim, S., Choi, K., Horisaki, R., and Brady, D. J., "Video-rate compressive holographic microscopic tomography," Opt. Express 19, 7289-7298 (Apr. 2011).

[30] Marim, M. M., Atlan, M., Angelini, E., and Olivo-Marin, J.-C., "Compressed sensing with off-axis frequencyshifting holography," Opt. Lett. 35, 871-873 (Mar. 2010).

[31] Cull, C. F., Wikner, D. A., Mait, J. N., Mattheiss, M., and Brady, D. J., "Millimeter-wave compressive holography," Appl. Opt. 49, E67-E82 (Jul 2010).

[32] Horisaki, R., Ogura, Y., Aino, M., and Tanida, J., "Single-shot phase imaging with a coded aperture," Opt. Lett. 39, 6466-6469 (Nov. 2014).

[33] Egami, R., Horisaki, R., Tian, L., and Tanida, J., "Relaxation of mask design for single-shot phase imaging with a coded aperture," Appl. Opt. 55, 1830-1837 (Mar. 2016).

[34] Raskar, R., Agrawal, A., and Tumblin, J., "Coded exposure photography: Motion deblurring using fluttered shutter," ACM Trans. Graph. 25, 795-804 (July 2006).

[35] Bub, G., Tecza, M., Helmes, M., Lee, P., and Kohl, P., "Temporal pixel multiplexing for simultaneous high-speed, high-resolution imaging," Nature Methods 7(3), 209-211 (2010).

[36] Gupta, M., Agrawal, A., Veeraraghavan, A., and Narasimhan, S. G., "Flexible voxels for motion-aware videography," in [Proceedings of European Conference on Computer Vision], 100-114 (2010).

[37] Reddy, D., Veeraraghavan, A., and Chellappa, R., "P2C2: Programmable pixel compressive camera for high speed imaging," in [Proc. IEEE Conf. Comp. Vision Pattern Recognition], 329-336 (June 2011).

[38] Liu, D., Gu, J., Hitomi, Y., Gupta, M., Mitsunaga, T., and Nayar, S. K., "Efficient space-time sampling with pixel-wise coded exposure for high-speed imaging," IEEE Trans. Pattern Anal. Mach. Intell. 36, 248-260 (Feb. 2014).

[39] Koller, R., Schmid, L., Matsuda, N., Niederberger, T., Spinoulas, L., Cossairt, O., Schuster, G., and Katsaggelos, A. K., "High spatio-temporal resolution video with compressed sensing," Opt. Express 23, 15992-16007 (June 2015).

[40] Llull, P., Liao, X., Yuan, X., Yang, J., Kittle, D., Carin, L., Sapiro, G., and Brady, D. J., "Coded aperture compressive temporal imaging," Opt. Express 21, 10526-10545 (May 2013).

[41] Wang, Z., Spinoulas, L., He, K., Tian, L., Cossairt, O., Katsaggelos, A. K., and Chen, H., "Compressive holographic video," Optics Express 25(1), 250-262 (2017).

[42] Ryu, D., Wang, Z., He, K., Zheng, G., Horstmeyer, R., and Cossairt, O., "Subsampled phase retrieval for temporal resolution enhancement in lensless on-chip holographic video," Biomed. Opt. Express 8, 1981-1995 (Mar 2017).

[43] Bioucas-Dias, J. M. and Figueiredo, M. A., "A new twist: two-step iterative shrinkage/thresholding algorithms for image restoration," IEEE Transactions on Image processing 16(12), 2992-3004 (2007).

[44] "DLP LightCrafter ${ }^{\mathrm{TM}}$ 4500." http://www.ti.com/lsds/ti/dlp/advanced-light-control/microarray-greaterthan-1million-lightcrafter4500.page. Accessed: 2016-06-01. 
[45] McElhinney, C. P., McDonald, J. B., Castro, A., Frauel, Y., Javidi, B., and Naughton, T. J., "Depthindependent segmentation of macroscopic three-dimensional objects encoded in single perspectives of digital holograms," Optics letters 32(10), 1229-1231 (2007).

[46] Mudanyali, O., Tseng, D., Oh, C., Isikman, S. O., Sencan, I., Bishara, W., Oztoprak, C., Seo, S., Khademhosseini, B., and Ozcan, A., "Compact, light-weight and cost-effective microscope based on lensless incoherent holography for telemedicine applications," Lab on a Chip 10(11), 1417-1428 (2010).

[47] Fienup, J. R., "Reconstruction of an object from the modulus of its fourier transform," Optics letters 3(1), 27-29 (1978).

[48] Fienup, J. R., "Phase retrieval algorithms: a comparison," Applied optics 21(15), 2758-2769 (1982).

[49] Marchesini, S., "Invited article: A unified evaluation of iterative projection algorithms for phase retrieval," Review of scientific instruments 78(1), 011301 (2007).

[50] Jaganathan, K., Eldar, Y. C., and Hassibi, B., "Phase retrieval: An overview of recent developments," arXiv preprint arXiv:1510.07713 (2015).

[51] Marchesini, S., He, H., Chapman, H. N., Hau-Riege, S. P., Noy, A., Howells, M. R., Weierstall, U., and Spence, J. C., "X-ray image reconstruction from a diffraction pattern alone," Physical Review B 68(14), 140101 (2003). 